\title{
NON SATURATION OF ENERGY IN A QUANTUM KICKED OSCILLATOR
}

\author{
M.V. DALY ${ }^{a}$ AND D.M. HefERnAN ${ }^{a, b}$ \\ ${ }^{a}$ Department of Mathematical Physics, St. Patrick's College \\ Maynooth, Co. Kildare, Ireland \\ ${ }^{b}$ School of Theoretical Physics, Dublin Institute for Advanced Studies, Dublin 4, Ireland
}

(Received November 28, 1995; revised version February 5, 1996)

\begin{abstract}
We present a quantum mapping for the kicked harmonic oscillator which relates the probability amplitudes of the undriven oscillator's eigenfunctions over successive kicks. We show how for various kick strengths the wave functions have a linear energy increase up to the limit imposed by the finite matrix size of the evolution matrix. We use this linear energy increase to define a quantum diffusion-like coefficient. We also show how this increase in energy causes the wave functions to spread out and become diffuse with little or no discernible structure. This model may serve as a paradigm for the study of quantum chaos.
\end{abstract}

PACS numbers: 03.65.-w, 05.45.+b

\section{Introduction}

One of the surprises of quantum chaos was the discovery of scars in the eigenstate spectrum of quantum systems which are classically unstable [1]. Heller postulated that they are remnants of the classical unstable periodic orbits (see Ref. [1] and references therein). In dissipative systems one can completely characterize chaos from a knowledge of the unstable periodic orbits $[2,3]$. In both classical and quantum chaos, knowledge of the orbit structure is fundamental and has been proved extremely useful in untangling and quantifying the dynamical properties of the system $[2,4]$.

The model we study is a fundamental one, both in classical and quantum physics $[5,6]$. The quantum version we study is more fundamental and complex than the kicked rotator. The kicked rotator in the classical limit exhibits chaos [7]. However in the quantum version of the problem the chaos is suppressed due to wave function localization similar to that of Anderson localization in amorphous materials $[8,9]$. Numerical analysis has shown the existence of a close relationship between saturation of diffusion in chaotic quantum systems and localization length 
of the wave functions (see Ref. [10] and references therein). The question arises whether the quantum suppression of chaos is generic. Indeed it has been argued that the saturation of diffusion due to quantum effects is not a manifestation of quantum chaos and may be present in more complicated models than the kicked rotator [11,12]. It has been postulated by Berman that the presence of an extra time scale $\left(\propto \ln \left(\hbar^{-1}\right)\right)$ in the quantum regime might allow for the possibility of the non suppression of chaos, that is the delocalization of the quantum wave functions and thus the non linearly kicked oscillator model would be a simple example where there would be quantum chaos [6]. It has become necessary to obtain a more detailed and quantitative understanding of the mechanism of diffusion in the quantum limit.

In this paper we present a quantum mapping which relates the probability amplitudes, $A_{m}(t)$, over successive kicks. The classical version of this quantum mapping is known to be chaotic with positive Lyapunov exponents and extensive K.A.M. tori breakup, with changes in parameters, in the classical map's stochastic layer [13]. It is our intention here to show how, using the quantum mapping, the calculated energy, $E(t)$, of the system has a linear-like increase which does not saturate within the time limits imposed by the finite size of the evolution matrix. Furthermore the time derivatives of these energies, $\partial E\left(t, \mu_{q}\right) / \partial t$, as a function of the kick strength, $\mu_{q}$, increases non linearly as the kick strength increases. In light of these results we aim to show that within the time limits imposed by the finite evolution matrix size the energy does not saturate and the wave functions are spreading out in an unbounded fashion.

\section{Quantum mapping}

The wave functions, $\psi(Q, t)$, for the kicked harmonic oscillator satisfies the time dependent Schrödinger wave equation given below

$$
\begin{aligned}
& \mathrm{i} \hbar \frac{\partial \psi}{\partial t}(Q, t)=\frac{1}{2}\left(\frac{\partial^{2}}{\partial Q^{2}}+Q^{2}\right) \psi(Q, t) \hbar \omega_{0} \\
& \quad+\mu_{q} \cos (K Q) \sum_{n=-\infty}^{\infty} \delta\left(t-n \frac{2 \pi}{\omega_{1}}\right) \psi(Q, t),
\end{aligned}
$$

where $Q$ is dimensionless position, $\mu_{q}$ is the strength of the kick term, $\omega_{0}$ and $\omega_{1}$ are the frequencies of the undriven oscillator and the kicks, respectively and $K$ is a dimensionless parameter determining the periodicity of the cos term. The wave functions, $\psi(Q, t)$, are composite being made up of a linear combination of the eigenfunctions, $u_{n}(Q)$, of the system with the time dependent coefficients $A_{n}(t)$

$$
\psi(q, t)=\sum_{n=1}^{\infty} A(t) u_{n}(Q),
$$

where the $u_{n}(Q)$ are the eigenfunctions of the undriven oscillator with the probability amplitudes, $A_{n}(t)$, relating the undriven oscillator's eigenfunctions to the driven composite wave functions, $\psi(Q, t)$, in Eq. (2). As expected the amplitudes are complex. The eigenfunctions for the undriven oscillator are [14]

$$
u_{n}(Q)=\frac{\mathrm{i}^{n}}{\sqrt{2^{n} \sqrt{\pi}(n !)}} H_{n}(Q) \exp \left(\frac{1}{2} Q^{2}\right)
$$


where $H_{n}(Q)$ are Hermite polynomials. The motivation behind this derivation stems from the well-documented existence of chaos in the classical limit [13] and the possibility of chaos existing in the quantum regime of this system as postulated by Berman et al. [6]. We now derive a mapping which relates the probability amplitudes from one kick to the next. Between successive kicks (from $t=(N \tau)^{+}$ to $t=((N+1) \tau)^{-}$for the $N$-th and $(N+1)$-th kick) the system behaves as the undriven system. Therefore any wave function $\psi(Q, t)$ evolves as

$$
\psi(Q, t)=\sum_{n=1}^{\infty} A_{n}(t) u_{n}(Q) \exp \left(-\mathrm{i} \frac{E_{n}}{\hbar} t\right),
$$

where $t$ is restricted to the time between kicks and the $E_{n}$ are the energy levels of the undriven system given by $(n+1 / 2) \hbar \omega_{0}$. Across any kick the wave function changes discontinuously by the amount $\mu_{q} \cos (K Q)$. Thus we can relate the wave functions from just before the $N$-th kick (at time $t=\left(N \tau^{-}\right)$) to just after it (at time $t=\left(N \tau^{+}\right)$) via the following identity:

$$
\psi\left(Q,(N \tau)^{+}\right)=\sum_{n=1}^{\infty} A_{n}(N \tau)^{-} u_{n}(Q) \exp \left(-\mathrm{i} \mu_{q} \cos (K Q)\right),
$$

where we have substituted for $\psi\left(Q,(N \tau)^{-}\right)$using Eq. (2). Using the identity (Eq. 8.511(4) in Ref. [15])

$$
\exp \left(\mathrm{i} \mu_{q} \cos (K Q)\right)=\sum_{s=-\infty}^{\infty} \mathrm{i}^{s} J_{s}\left(\mu_{q}\right) \exp (\mathrm{i} s K Q),
$$

where $J_{s}$ is the $s$-th Bessel function of first kind, we can relate the evolution of the wave functions over successive kicks

$$
\begin{aligned}
& \psi\left(Q,((N+1) \tau)^{-}\right)=\sum_{p=1}^{\infty} A_{p}\left((N \tau)^{-}\right) u_{p}(Q) \sum_{s=-\infty}^{\infty} \mathrm{i}^{s} J_{s}\left(\mu_{q}\right) \\
& \quad \times \exp (\mathrm{i} s K Q) \exp \left[-\mathrm{i}\left(p+\frac{1}{2}\right) \omega_{0} \tau\right] .
\end{aligned}
$$

If we substitute $\sum_{r=1}^{r=\infty} A_{r}\left(((N+1) \tau)^{-}\right) u_{r}(Q)$ for the $\psi\left(Q,((N+1) \tau)^{-}\right)$and then take the expectation value of each side of the expression in Eq. (6) with $u_{m}(Q)$, we can relate each of the probability amplitudes at the later time, $A_{m}\left(((N+1) \tau)^{-}\right)$, to those at the earlier time, $A_{p}\left((N \tau)^{-}\right)$, by varying $m$ over all the eigenfunctions, $u_{m}(Q)$; i.e.

$$
\begin{aligned}
& A_{m}\left(((N+1) \tau)^{-}\right)=\sum_{p=1}^{\infty} A_{p}\left((N \tau)^{-}\right) \\
& \quad \times \sum_{s=-\infty}^{\infty} \mathrm{i}^{s} J_{s}\left(\mu_{q}\right) \exp \left[-\mathrm{i}\left(p+\frac{1}{2}\right) \omega_{0} \tau\right]\left\langle u_{m}(Q) \mid u_{p}(Q) \exp (\mathrm{i} s K Q)\right\rangle .
\end{aligned}
$$

The bra-ket term above can be calculated by evaluating an integral over the Hermite polynomials constituting the $u_{m}(Q)$ and $u_{p}(Q)$. Using the identity (Eq. 7.374(7) in Ref. [15])

$$
\int_{\infty}^{\infty} \exp \left[-(x-y)^{2}\right] H_{m}(x) H_{p}(x) \mathrm{d} x=2^{p} \sqrt{\pi}(m !) y^{p-m} L_{m}^{p-m}\left(-2 y^{2}\right)
$$


which is valid for $m \leq p$ we find that our expression for the probability amplitudes in Eq. (7) becomes

$$
\begin{gathered}
A_{m}\left(((N+1) \tau)^{-}\right)=\sum_{p=m}^{\infty} A_{p}\left((N \tau)^{-}\right) \sum_{s=-\infty}^{\infty} \mathrm{i}^{s} I_{s}\left(\mu_{q}\right) \exp \left[-\mathrm{i}\left(p+\frac{1}{2}\right) \omega_{0} \tau\right] \\
\times \frac{\mathrm{i}^{m+p}}{\sqrt{\pi 2^{m+p}(p !)(m !)}} \exp \left(-s^{2} K^{2}\right) 2^{p} \sqrt{\pi}(m !)(\mathrm{i} s K)^{p-m} L_{m}^{p-m}\left(s^{2} K^{2}\right),
\end{gathered}
$$

which is valid for $m \leq p$. However, the $H_{m}(x)$ and $H_{p}(x)$ in Eq. (8) can be swapped giving us a complementary expression for the case $p \leq m$ which is identical to Eq. (9) except for the substitution of $m$ for $p$. This fact is very important as we will now show. Upon examination of Eq. (9), the only term which depends on time on the right hand side is $A_{p}$. All the others are time independent allowing us to group them together as a single time invariant term which we call $U$

$$
A_{m}\left(((N+1) \tau)^{-}\right)=\sum_{p=1}^{\infty} U_{m p} A_{p}\left((N \tau)^{-}\right) .
$$

The term $U_{m p}$ is the $m p$ element of the infinite time-independent square matrix, $U$, which evolves the system forward in time. Furthermore, as we can interchange the $m$ and $p$ in Eq. (9) above to evaluate the case when $p \leq m$, one can easily check that the matrix is symmetric (i.e the transpose of $U$ is $U$ )

$$
U_{m p}=U_{p m} \text {. }
$$

We refer to $U$ as the time evolution matrix of the system. Knowing the time evolution of the amplitudes allows us to calculate the time evolution of the system's wave functions, $\psi_{t}$, and the total energy of the system, $E$. Thus, for any wave function, $\psi$, we calculate the energy to be

$$
E_{\psi}\left((N \tau)^{-}\right)=\sum_{r=1}^{\infty}\left\|A_{r}\left((N \tau)^{-}\right)\right\|^{2}\left(r+\frac{1}{2}\right) \hbar \omega_{0} .
$$

The numerical work to calculate the matrix $U$ relied on the truncation of the summation over $s$ in Eq. (7) brought about by the exponential term, $\exp \left(-s^{2} K^{2}\right)$, and the Bessel functions. This truncation enabled the matrix to be numerically evaluated. Once calculated this matrix, $U$ could be used for the parameter set which gave rise to it for any time length up to and including the finite size of the matrix calculated.

\section{Results}

The initial distribution of the probability amplitudes, $A_{m}(0)$, is a mixed parity state having a gaussian profile of the amplitudes, centred on $m=10$. We chose a low value of $m$ primarily because of the size limitations on our calculated evolution matrix $U$. The evolution matrix $U$ was restricted to a $350 \times 350$ matrix with the implication that, any state which spreads, or diffuses, so that the amplitudes near the $m=350$ boundary are becoming increasingly significant, there is an artificial time limit imposed beyond which the results are boundary influenced and hence untrustworthy. 

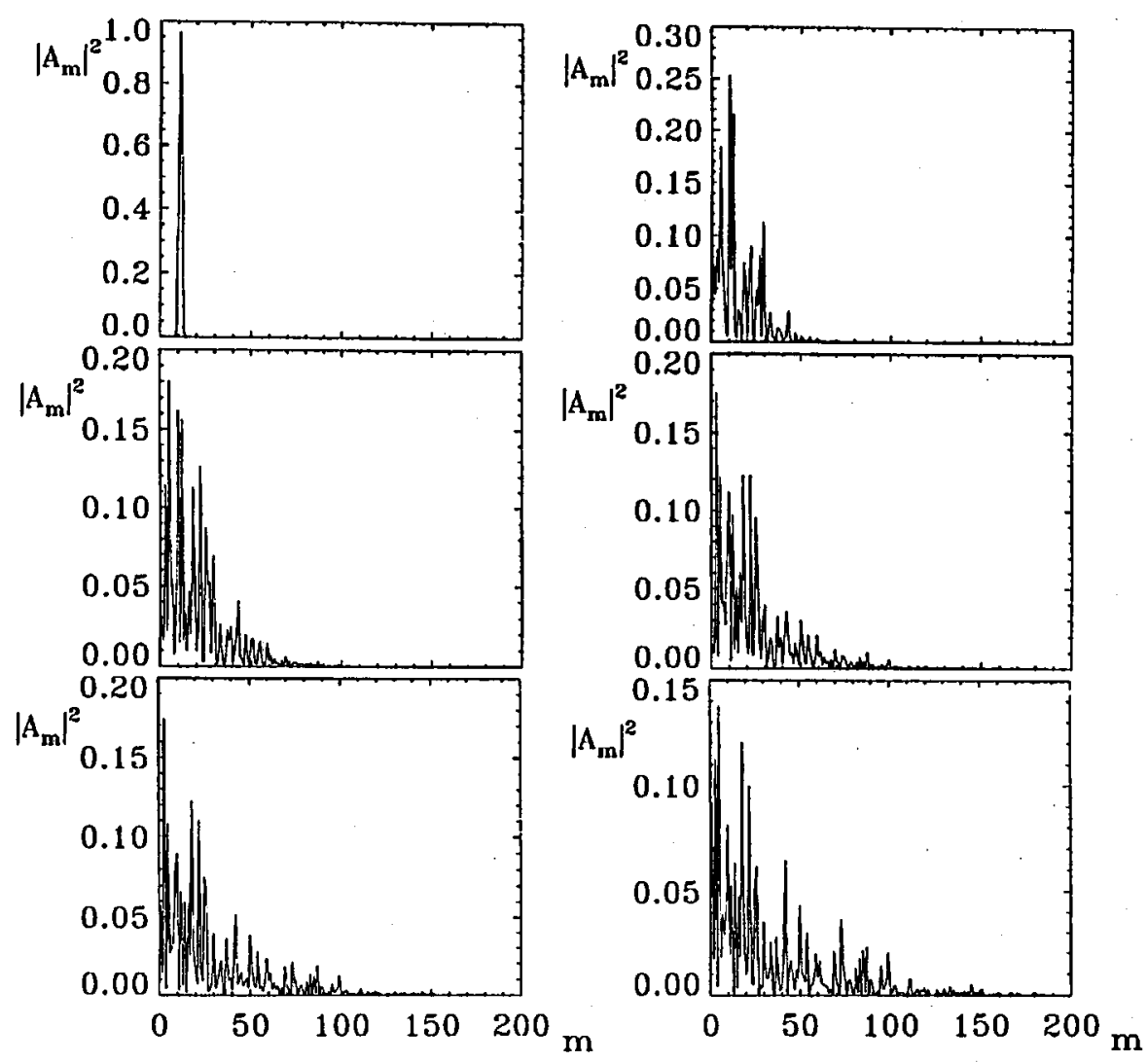

Fig. 1. The amplitude spectrum for the initial mixed parity state centred at $m=10$. $\mu_{q}=1.5$ and the time between successive plots is 4 time steps. The spread over the states is apparent and boundary saturation occurs soon after (f) at around 40 time steps.

In Fig. 1 we present the case for the quantum mapping when $\beta=\pi / 2$ and $\mu_{q}=1.5$. It is apparent that the initial distribution of amplitudes rapidly becomes spread out over the complete subset of amplitudes calculated for the evolution matrix, $U$. The spreading was so rapid that, just over forty iterations after the initial state, the calculations were stopped as the upper boundary of the probability amplitudes had been reached. The following condition was used to stop the calculations: if the magnitude of the amplitude for $m=340$ was greater than $10^{-30}$ then the calculation stopped. Thus the magnitudes of the amplitudes above 340 were non-contributory. In fact one can see from the results presented in Fig. 1 that $m$ is not shown above 200 because of its small size. Larger matrices should provide better long term behaviour and this is being pursued by considering the behaviour of individual elements in the matrix itself. The principle diagonal is the dominant part of the evolution matrix with the off diagonal terms falling off rapidly each side of this principle diagonal. The rapidity of fall off is naturally inversely 
dependent on the size of the kick strength $\mu_{q}$ and, for the range of kick strengths considered in this article, were found to drop below 5 orders of magnitude within 50 elements of the principle diagonal. This allows us to evaluate larger arrays by just evaluating a tract of 100 elements wide centred on the principle diagonal instead of half the $n \times n$ matrix (the symmetry of the matrix necessitates calculation of only half of the matrix).

In Fig. 2 we present the probability amplitudes for the corresponding case in Fig. 1. These plots show the system at the corresponding times to those in Fig. 1 and show the rapid spreading inherent in the system for the chosen set of values. What is also apparent from these six plots is the rapid onset of diffusivity in the probability amplitudes as time increases. To further analyse the system for this kick strength and for kick strengths close to this value, we calculated the energy
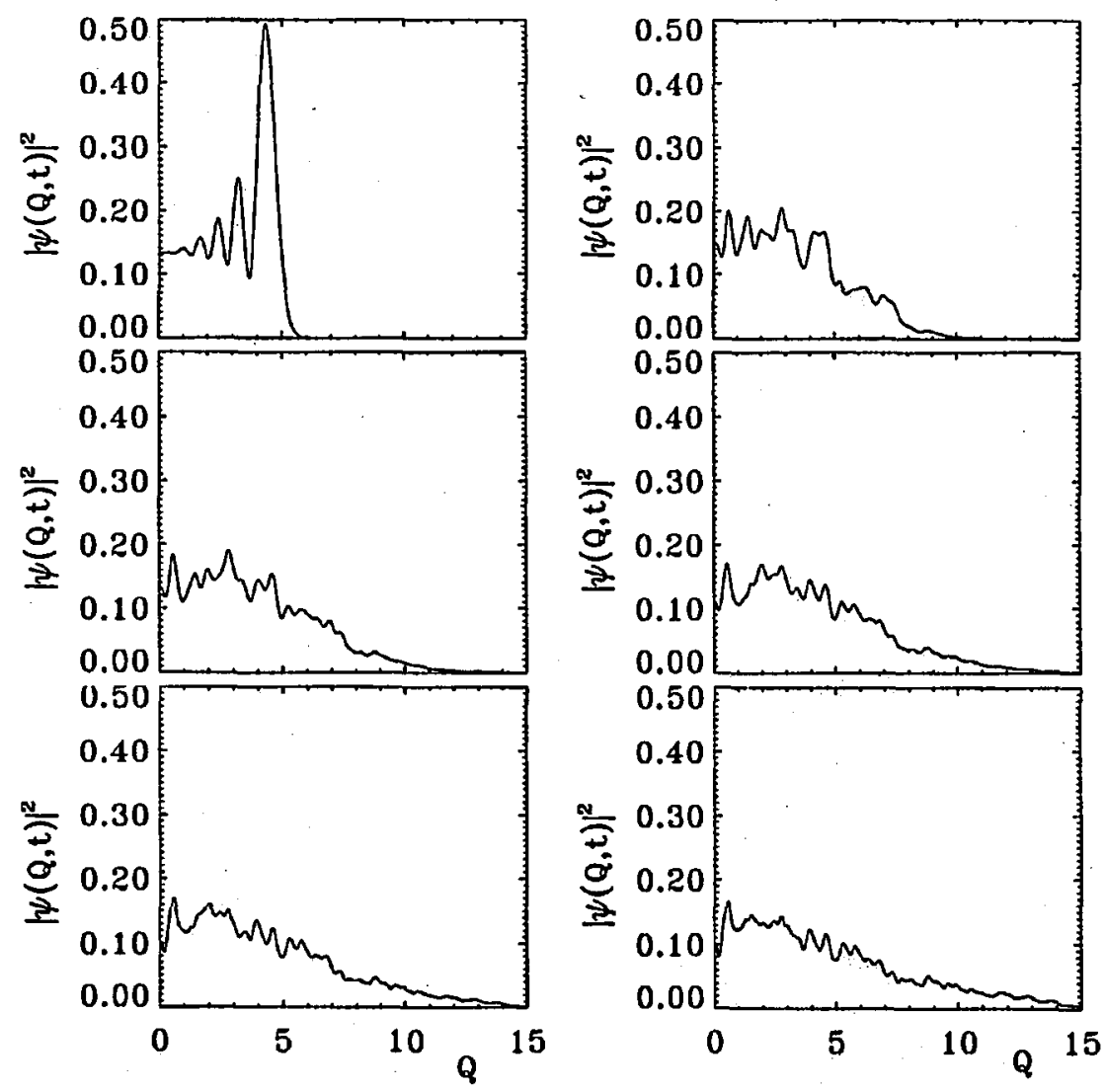

Fig. 2. The probability amplitudes, $|\psi(Q, t)|^{2}$, corresponding to the parameters of Fig. 1 above. The time between each plot is 6 iterations of the mapping. Even over such a short period of time the spread over $Q$ and the increasing lack of structure indicates a non-saturating behaviour for this state. 
of the system as a function of time. This serves as an indicator for localised or de-localised behaviour in the system. In Fig. 3 we give a composite energy plot of five different kick strengths over a specified time. The linear nature of these energy curves suggests it might be possible to evaluate a quantum diffusion coefficient, $D_{q}\left(\mu_{q}\right)$. There is a number of problems associated with this exercise including the small time interval over which the energy does not saturate by impinging on the boundary, the requirement to calculate a new evolution matrix for each value of the kick strength and the need to average over a set of states which is further limited by the finite size of the unitary matrices. However, we have calculated a number of matrices of size $350 \times 350$ and for these matrices the energy increases linearly and we are able to calculate a quantum diffusion coefficient, $D_{q}\left(\mu_{q}\right)$, which we define as

$$
D_{q}\left(\mu_{q_{0}}\right)=\left\{\frac{\partial E\left(\mu_{q}, t\right)}{\partial t}\right\}_{\mu_{q} \equiv \mu_{q_{0}}} .
$$

The result is presented in Fig. 4. There is an enhancement at $\mu_{q}=1.5$. However, at finer resolution we found the neighbouring points to be well behaved (in the sense they followed the general trend of the curve) but the slope of the energy at $\mu_{q}=1.5$ took a definite jump. This occurrance at close to $\pi / 2$ cannot be discarded and may be a kind of enhancement similar to the classical enhanced diffusion due to accelerator islands [16]).
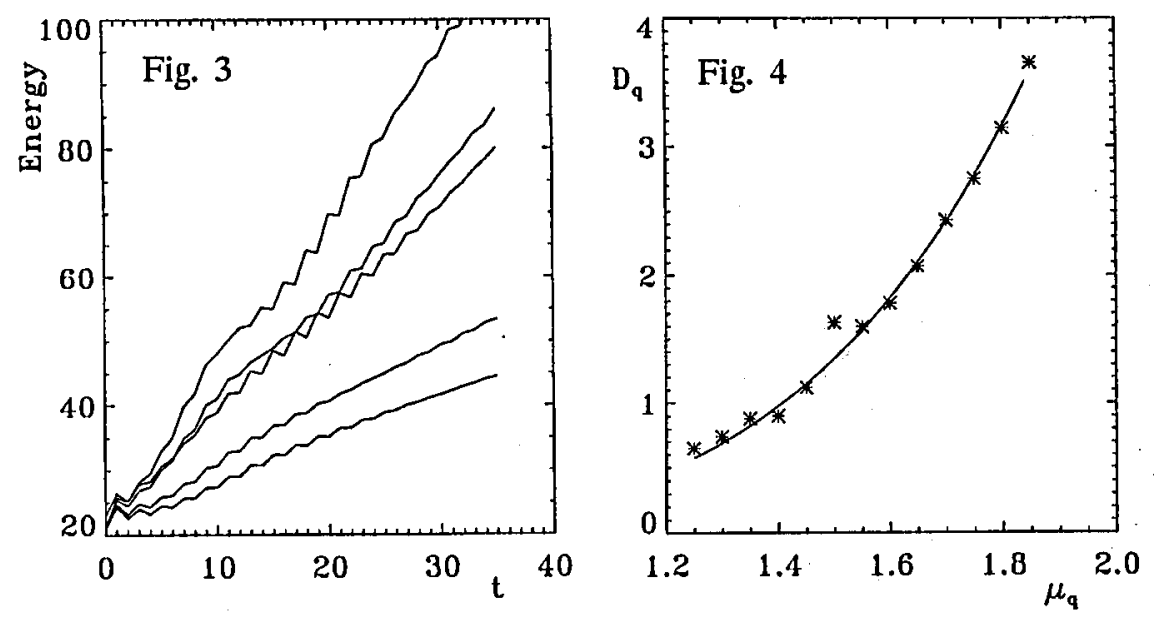

Fig. 3. Five energy curves for the mixed state at $m=10$. The curves correspond to (from top to bottom) $\mu_{q}=1.7,1.6,1.5,1.4$ and 1.3. The linear type nature of these curves prompted us to calculate the rate of energy increase in time (a sort of quantum diffusion coefficient) denoted $D_{q}$ for each value shown.

Fig. 4. The coefficient, $D_{q} \equiv \partial E(t) / \partial t$, vs. $\mu_{q}$. The increase was found generally to increase as $A\left(\mu_{q}\right)^{b}$ with $A=0.2$ and $b=4.68$. The solid line is the fit associated with the $A$ and $b$. 
It could be argued that the time taken for the saturation to occur is not long enough to be conclusive of an energy saturation. Indeed taken alone such time evolutions are not capable of proving the energy will never saturate or that the wave functions will never become localised. What did in fact convince us of the possible existence of delocalised wave functions and a continued increase in the energy evolution was the calculated Wigner distribution [17], $P_{\mathrm{W}}(Q, P)$, (or quasi-phase space) at various time steps for the given kick strength. The distribution evolved from its initial mixed state to a state whose quasi-phase space had a striking four-fold symmetry reminiscent of the tiled structure seen in the classical phase space for the value of $\beta$ chosen (i.e. $\beta=\pi / 2$ ) (see Ref. [13]).

For the classical oscillator on resonance and chaotic, the stochastic layer has a crystalline (or quasi-crystalline) structure depending on the ratio $\omega_{0} / \omega_{1}=$ $n / m$ with $n, m \in Z$. The Wigner distribution is shown in Figs. 5a and 5b, it is finite in size because the upper limit imposed on the calculation by the finite matrix size limits the highest order eigenfunction used in the calculation which in turn limits the extent of the distribution. As time progresses we expect the wave functions to continue spreading over the undriven system's eigenfunctions thus rendering a continued extended tiled structure in the quasi-phase space of the Wigner distribution. The beginnings of this extended structure can be seen by examining Fig. 5a. The corners of the inner $3 \times 3$ square region in the distribution, though truncated due to the finite array size, clearly extend beyond the region given whereas at the top, left, bottom and right middle positions the edge of a new set of islands is also apparent. Furthermore, this tiled structure with its

(a)

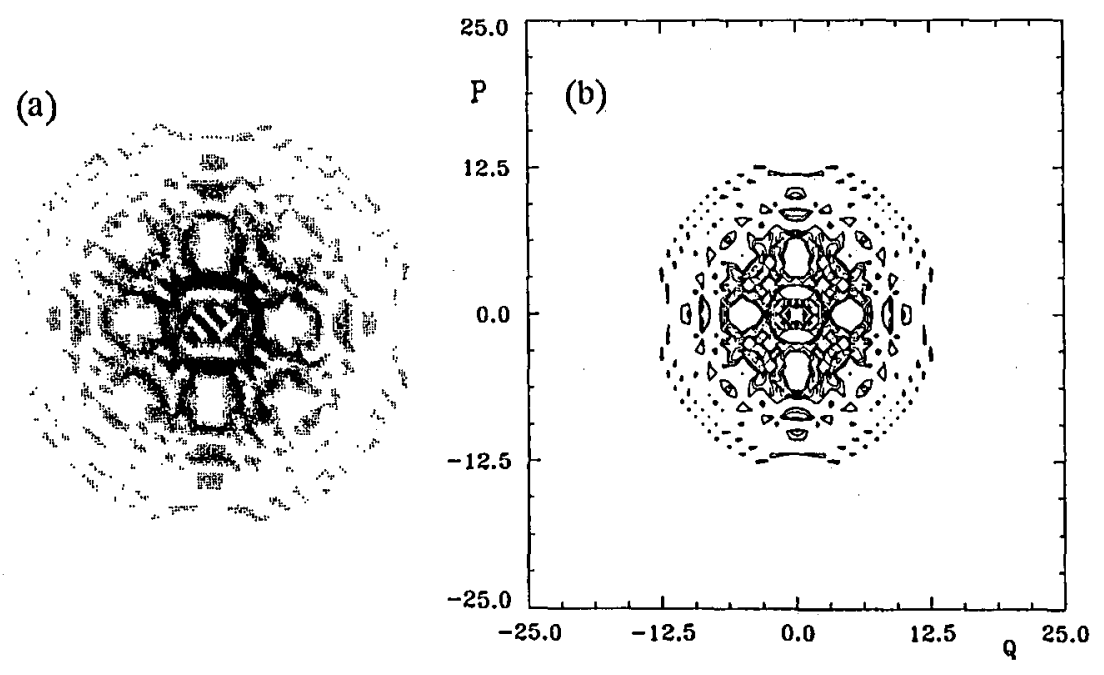

Fig. 5. The Wigner distribution, $P_{\mathrm{w}}(Q, P)$ after 20 time steps. The system is on resonance with $\beta=\pi / 2$ and $\mu_{q}=1.5$. Figure (a) shows an image of the distribution with the dominant 4 -fold symmetry being very evident. Figure (b) shows the corresponding contour plot of the distribution. The extended structure is also evident. 
clearly defined circular islands indicates that some orbits may exist in this system which have significant overlap with the regions between the islands which in the classical regime corresponds to the stochastic layer region of unbounded, diffuse transport. Furthermore these orbits could be spreading out rapidly if the influence of these inter-island regions in the Wigner distribution is directly comparable to the classical stochastic layer.

\section{Conclusions}

We have derived a mapping for the quantum non linearly kicked harmonic oscillator which maps the probability amplitudes at each time step to the set of probability amplitudes at the previous time step. Using this mapping we have shown the existence of states whose time dependence on energy is linear and for whom a diffusion-like coefficient can be defined which increases non linearly with the kick strength, $\mu_{q}$. These results, coupled with the structure found in the quasi-phase space of the Wigner distribution for the system, seem to indicate the existence of states in this system whose energy does not saturate and the possibility of delocalised orbits which spread out rapidly along an apparent quantum version of the classical phase space stochastic layer.

\section{Acknowledgment}

This work is funded by Forbairt, the Irish National Agency for Science and Technology.

\section{References}

[1] E.J. Heller, in: Chaos and Quantum Physics, Eds. M.J. Giannoni, A. Voros, J. ZinnJustin, North-Holland, Amsterdam 1991, p. 547.

[2] D. Auerbach, P. Cvitanovic, J. Eckmann, G. Gunaratne, I. Procaccia, Phys. Rev. Lett. 58, 2387 (1987).

[3] M. Feigenbaum, M. Jensen, I. Procaccia, Phys. Rev. Lett. 56, 1503 (1986).

[4] M. Gutzwiller, Chaos in Classical and Quantum Mechanics, Springer-Verlag, New York 1990.

[5] A.A. Chernikov, R.Z. Sagdeev, D.A. Usikov, G.M. Zaslavsky, Sov. Sci. Rev. C. Math. Phys. 8, 83 (1989).

[6] G.P. Berman, V.Yu. Rubaev, G.M. Zaslavsky, Nonlinearity 4, 543 (1991).

[7] G. Casati, B.V. Chirikov, F.M. Israilev, J. Ford, in: Stochastic Behaviour in Classical and Quantum Hamiltonian Systems, Eds. G. Casati, J. Ford, in series Lecture Notes in Physics, Vol. 93, Springer, Berlin 1979, p. 334.

[8] S. Fishman, D.R. Grempel, R.E. Prange, Phys. Rev. Lett. 49, 509 (1984).

[9] D.R. Grempel, R.E. Prange, S. Fishman, Phys. Rev. A 29, 1639 (1984).

[10] F.M. Israilev, Phys. Rep. 106, 299 (1990).

[11] D. Shepelyansly, Physica D 8, 208 (1983).

[12] S. Adachi, M. Toda, K. Ikeda, Phys. Rev. Lett. 61, 659 (1988).

[13] M.V. Daly, D.M. Heffernan, J. Phys. A 28, 2515 (1995).

[14] A. Messiah, Quantum Mechanics, Vol. 1, North-Holland, Amsterdam 1976. 
[15] I.S. Gradshteyn, I.M. Ryzhik, Table of Integrals, Series and Products, 4ed., Academic Press, New York 1965.

[16] M. Ishizaki, T. Ilorita, H. Mori, Prog. Theor. Phys. 85, 1013 (1991).

[17] E. Wigner, Phys. Rev. 40, 749 (1932). 